\title{
Educación integral para el éxito profesional del Administrador
}

\section{Integral education for the professional success of the Administrator}

Guadalupe Morales-Mejía'; Falinda-Elizabeth Bahena-Benítez²

\section{Palabras claves:}

Educación, Estudiantes, Competencias Calidad

Artículo de investigación:

Fecha de recepción: 2019/08/17

Fecha de aceptación: 2019/12/16

Esta publicación se encuentra bajo licencia:

Creative Commons ReconocimientoNoComercialSinObraDerivada 4.0 Internacional

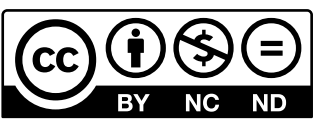

\section{Resumen}

La educación en México ha sido objeto de estudio por décadas, en medios como congresos internacionales, políticas publicas, planes y programas de gobierno que han determinando la importancia que tiene en la enseñanza y trascedencia para la formación de los alumnos de educaciòn básica, articulada con los programas educativos que conllevan a la educación integral, vista como la formación de los aspectos: intelectual, emocional, social, físico, que fortalezcan a los chicos y de esta forma adquieran las competencias y valores para enfrentar una sociedad exigente y competitiva como profesionistas. La investigación se realizó en 3 escuelas primarias de la ciudad de Puebla, todas coincidieron en que los estudiantes egresan con bajo rendimiento académico, con falta de valores y deficientes destrezas, por lo que al llegar a la Universidad donde los recibimos presentan debilidades en los conocimientos, compromiso, responsabilidad. Por lo tanto, es necesario implementar un modelo de Educación Integral con el que se obtengan los conocimientos y habilidades necesarias que a su vez los motivaría para ingresar a la Universidad y así mismo egresarían con las competencias que les permita un mejor desempeño como profesonistas para ocupar un lugar en las MiPyMES que representan el 90\% de las

1 Licenciada en Administración Pública, Doctora en Administración Pública, Profesor Investigador Titular tiempo completo, México, samy92lu@gmail. com, tosa8992@hotmail.com, código ORCID: https://orcid.org/0000-0003$1422-6580$

2 Profesor Investigador Asociado C tiempo completo, Contador Público, Doctora en ciencias de la Educación, México, faly_eder@hotmail.com, código ORCID: https://orcid.org/0000-0002-7014-6166 


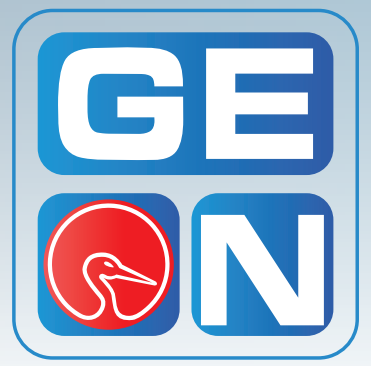

Revista GEON

(Gestión, Organizaciones y Negocios)

ISSN: 2346-3910 en línea

revistageon@unillanos.edu.co

Universidad de los Llanos

Colombia

Morales Mejía, G., \& Bahena Benítez, F. (2020).

Educación integral para el éxito profesional del Administrador

Revista GEON

(Gestión, Organizaciones y Negocios), 7(1), 83-95.

\section{도키}

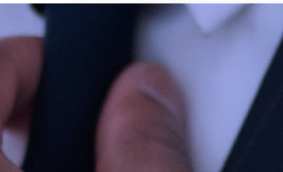

66 es necesario implementar un modelo de Educación Integral con el que se obtengan los conocimientos y habilidades necesarias que a su vez los motivaría para ingresar a la Universidad y así mismo egresarían con las competencias que les permita un mejor desempeño

Morales Mejía, G., \& Bahena Benítez, F. (2020):

Educación integral para el éxito profesional del

Administrador. Revista GEON (Gestión,

Organizaciones Y Negocios), 7(1), 83-95.

http://revistageon.unillanos.edu.co 
Empresas en Puebla y generan el $72 \%$ del empleo (INEGI 2017) siendo la fuente de subsistencia para la sociedad Poblana.

Palabras claves: Educación, Estudiantes, , Competencias Calidad

\section{Abstract}

Education in Mexico has been the subject of study for decades, in media such as international congresses, public policies, plans and government programs that have determined the importance it has in teaching and transcendence for the training of students in basic education articulated with educational programs that lead to integral education, seen as the formation of aspects: intellectual, emotional, social, physical, that strengthen children and thus acquire the skills and values to face a demanding and competitive society as professionals. The research was conducted in 3 primary schools in the city of Puebla, all agreed that students graduate with low academic performance, lack of values and poor skills, so when they arrive at the University where we receive them, they present weaknesses in knowledge, commitment, responsibility. Therefore it is necessary to implement a model of Integral Education with which the necessary knowledge and skills are obtained that in turn would motivate them to enter the University and likewise would graduate with the skills that allow them a better performance as professionals to occupy a place in the MSMEs that represent $90 \%$ of the Companies in Puebla and generate 72\% of employment (INEGI 2017) being the source of subsistence for the Poblana society.

Keywords: Education, Students, Development, Skills Quality 


\section{Introducción}

La educación integral es un modelo de enseñanza aprendizaje cuyo propósito es lograr una educación que cubra los aspectos necesarios para dar seguridad, fortalecer el compromiso, la responsabilidad, los valores, los conocimientos y habilidades tales como: capacidad de adaptación, control de estrés, capacidad de comunicación, innovación y creatividad, toma de decisiones para los egresados del nivel superior. En México enfrentamos escenarios que están cuestionando la educación tradicional donde se enseña en un espacio reducido, con métodos que solo permiten al estudiante manejar conocimientos elementales, donde solo el profesor y el alumno participan en la mayoría de los casos.

Esta situación abre espacios de reflexión y análisis para plantear alternativas que conlleven a otro contexto, algunas de estas evoluciones son impulsadas por diversas propuestas de las políticas educativas establecidas por el estado mexicano. El objeto de la investigación es relacionar el egreso de los niños de educación básica, con el ingreso de los jóvenes de educación superior, realizando un diagnòstico para conocer las habilidades de los estudiantes, por lo tanto, dicha investigación se llevo a cabo en tres escuelas de la zona sur de la ciudad de Puebla, se realizo con base en una muestra de conveniencia, tomando en cuenta la ubicación, y que contaran con la organización formal para lograr los resultados esperados.

\section{Contexto teórico}

Es indudable que la educación tiene una función social que puede ser aprovechada para corregir estereotipos de género. Émile Durkheim, argumenta que la educación como medio de transmisión de ideas y valores, no solo debe concentrarse en el acceso al derecho, sino también en el contenido de lo que se transmite, la implementación de programas educativos especialmente dirigidos a combatir la discriminación contra las mujeres puede servir para cambiar ideas profundamente arraigadas en la sociedad. (Cornejo, M 2018) en temas organizacionales Gary Hamel Citado por Leal Cèspedes (2013) nos menciona que es necesario que las empresas se transformen de acuerdo a como el mundo se desarrolla.

Es necesario aclarar que, el valor humano depende en lo fundamental del medio social en que se desenvuelve el hombre, porque se relaciona con la forma en que se asimilan esas condiciones sociales, capacitándolo para que responda al medio social en el que vive relacionado con los valores para ello un plan de entrenamiento organizado desarrollará las competencias necesarias en las personas (Torres-Flòrez, 2019).

El papel de la educación parece haberse consolidado a partir de su capacidad para: facilitar el acceso a trabajos inteligentes, a redes de conocimiento, minimizar desigualdades, aportar elementos críticos para interpretar la realidad incorporando el componente multicultural y construir ciudadanía en contextos democráticos. En este sentido, han avanzado las reformas educativas que se ejecutaron en casi todos los países de América Latina y que, sin embargo, 
no consiguieron eliminar algunos de los factores que determinan un alto grado de estratificación y consecuentemente de inequidad, al interior de sus sistemas educativos.

La transformación en la educación es un factor fundamental para que los profesores y estudiantes reúnan las habilidades, capacidades, creatividad integrando los conocimientos y valores necesarios para enfrentar los retos del presente y del futuro, para alcanzar altos niveles de competitividad (Innov. educ. 2017) Es forjar individuos, capaces de una autonomía intelectual y moral y que respeten esa autonomía del prójimo, en virtud precisamente de la regla de la reciprocidad.

Estos conceptos no se aplican extensamente si analizamos resultados y evaluaciones, la pregunta es ¿cuáles son los resultados?, cual es el problema si lo hay, que pasa con los profesores, los alumnos, los programas, el método o la técnica, pues bien me ha interesado desde siempre apoyar a los estudiantes en su desarrollo y desempeño académico conocer lo que sucede y de acuerdo a la evaluación el $15 \%$ de los chicos tiene dificultades para leer, para estudiar, para investigar, las habilidades y competencias son escasas por lo que me di la tarea de realizar la investigación e indagar cuales son los factores que no permiten el desarrollo del aprendizaje en los estudiantes, lamentablemente coincidieron en cinco factores:

\section{Pobreza familiar}

2. Desintegración familiar

3. Carencia de valores

\section{Inadecuada alimentación \\ 5. Desigualdad de oportunidades}

Para explicar estas diferencias es necesario analizar los factores que determinan la falta de competencias y las diferencias en las condiciones socio-culturales además de los aspectos económicos de los estudiantes y de los profesores. Por lo tanto es considerable establecer y aplicar un modelo de educación integral y que es la educación integral, es una filosofía educacional y forma constructivista basada en la premisa de que toda persona encuentra su identidad, y el significado y sentido de su vida, a través de nexos con la comunidad, el mundo natural, y los valores. Se trata de una educación completa e integradora, que busca despertar una devoción intrínseca por la vida y la pasión por el aprendizaje. (Wompner, F2019).

Cada periodo de Gobierno se impulsan políticas educativas con propuestas de cambios estructurales que exigen tiempo y compromiso de los actores que son: los directivos, los padres de familia, los estudiantes, la institución, para llevarse a cabo la implementación de estas políticas debe luchar con la complejidad y diversidad de un sistema educativo Méxicano.

Si se habla de Educación se debe defender una concepción de la formación más vinculada al servicio Público y el articulo tercero Constitucional, es decir un conjunto de políticas que tienen como fin último la mejora de las condiciones de vida del individuo.

Considerando lo anterior, falta mucho por hacer para lograr un crecimiento 
y desarrollo que sea consecuencia de los objetivos de las políticas educativas. El estudio de campo se realizo en las siguientes escuelas:

\section{Escuela 1}

Primaria oficial turno matutino, Institución, Profesora Amalia Contreras de Lobato C.C.T.21EPRO723M de la zona urbana del municipio de Puebla Zona escolar 043 en la que solo se imparte Educación básica por ahora y las observaciones son las siguientes: nivel socioeconómico medio bajo, cuenta con 36 aulas de clase y 36 profesores

\section{Escuela 2}

Primaria Federal Justo Sierra CCT: 21DPR18930 de la ciudad de Puebla, se imparte educación básica, cuyo nivel socioeconómico es bajo. Cuenta con 13 aulas y 15 profesores.

\section{Escuela 3}

Centro escolar comunitario del Sur Lic. Manuel Bartlett Díaz, se imparte educación básica, educación secundaria y bachillerato Cuenta con 23 profesores 20 aulas, cuenta con área deportiva, sala de cómputo, áreas verdes.

La presente investigación se llevó a cabo con la participación de las autoridades escolares de, con el fin de obtener la información necesaria para conocer la realidad vivida en dichas instituciones.

Para poder acceder a la vida de dichas escuelas, es necesario conocer acerca de su contexto, tanto interno como externo, con la finalidad de ubicar la investigación en el escenario real en el que se llevó a cabo, el cual se describe a continuación. Las primarias con las que se trabajó son identificadas como: Escuela 1, 2 y 3 pertenecen a esta misma zona escolar, por lo cual comparten algunas de sus características. Las tres son instituciones urbanas públicas y de organización formal, es decir, cuentan con un director y maestros frente a cada uno de los grupos, ofreciendo los seis grados correspondientes a la educación primaria. Además, se encuentran en zonas urbanizadas de la ciudad, contando con todos los servicios públicos y atendiendo, en su mayoría, a alumnos de clase media baja.

La Escuela 1 está ubicada, en una zona habitacional. La directora de esta escuela funge como tal desde hace 3 años. El personal está conformado por 36 profesores frente a grupo, de los cuales 7 son varones y 29 son mujeres, quienes tienen en promedio 10 años laborando en la institución. Además, se cuenta con dos docentes que tienen el cargo de Asesor Técnico Pedagógico (ATP), asistiendo a las funciones directivas y de lectura respectivamente, 3 profesores de educación física, y 8 intendentes. Actualmente, se atiende a un promedio de 495 alumnos, contando con dos grupos de cada grado escolar.

La Escuela 2 se ubica en la ciudad d Puebla. El director tiene el cargo desde hace 4 años. La institución cuenta con una planta docente completa, conformada por 15 profesores frente a grupo, de los cuales 5 son varones 
y 10 son mujeres, quienes tienen en promedio 12 años de laborar en esta misma escuela. Además, se cuenta con cuatro docentes que ocupan el cargo de ATP en las áreas de salud, lectura y asistencia a la dirección, 1 profesores de educación física, una persona encargada del aula de medios, y 3 intendentes.

De manera regular, en la escuela se llevan a cabo programas extracurriculares con el objetivo de beneficiar la formación de los alumnos.

En cuanto a las instalaciones físicas del plantel, se cuenta con 15 salones equipados con pizarrón, mesa-bancos, loseta y persianas. También se tiene un aula con equipo de cómputo y audiovisual al servicio de los docentes y los alumnos de todos los grados, conocida como "aula de medios", una cooperativa en donde se venden alimentos a los estudiantes, dos canchas deportivas y un almacén.

La Escuela 3 Cuenta con 20 aulas, 23 profesores, 797 alumnos, cuenta con área deportiva, sala de cómputo, áreas verdes, instalaciones de luz, agua potable, baños. También se pudo observar inseguridad,

El nivel socioeconómico de los alumnos es medio bajo, lo que se manifiesta por la vestimenta que utilizan los alumnos que asisten a la escuela y la dificultad para llevar los materiales que se les piden. Los alumnos Ilegan a la escuela caminando o en transporte publico, porque viven cerca de esta.

Durante la investigación, se analizó la dinámica de las tres escuelas primarias públicas descritas en el aparta- do anterior, como ya se aclaró, todas están en el área urbana del municipio de Puebla, participantes vigentes en el programa escuelas de calidad (PEC), con el fin de que las tres escuelas se encuentren dentro del mismo contexto

La selección de las escuelas se hizo con base en una muestra de conveniencia, se realizaron visitas exploratorias en el mes de julio, septiembre y noviembre 2019, para contar con la descripción del contexto de manera previa a las observaciones, que se realizaron.

Durante el trabajo de campo del presente estudio, se contó con la participación de distintos actores de la comunidad escolar del municipio, con los cuales se abordaron distintas técnicas de recolección de datos descritas anteriormente.

Cabe señalar que las reuniones de los directores siempre fueron de carácter individual. En el mes de Julio 2019, se inició la aplicación de los instrumentos diseñados para la recopilación de información del estudio. Durante este mes se realizaron las 2 visitas de observación no participante, en cada una de las instituciones. Durante el mes de septiembre y noviembre se realizaron el resto de las actividades, consistentes en las dos visitas de observación no participante a las escuelas.

Con el propósito de recabar la información necesaria para el cumplimiento de lo planeado, se determinó poner en práctica dos técnicas básicas de recolección de datos: observación y entrevista a continuación se describen las características de cada 
una de las técnicas utilizadas, además de los instrumentos que se aplicaron.

Se realizó una estancia de tres días en cada escuela, con el objetivo de recuperar información a través de la observación no participante, entendida como la técnica en la que "un plan aplico la guía de entrevista previamente preparada y concentra la atención en ciertos aspectos de la conducta, sin interacción entre el observador y el sujeto o grupo observado

La interpretación posterior de las anotaciones registradas en el diario de campo, se describieron y tuvieron como insumo la experiencia obtenida en la visita de observación exploratoria a cada escuela, realizada en el mes de Julio y septiembre y noviembre 2019.

Durante la observación se llevó a cabo el registro de manera sistemática las acciones de los profesores y alumnos de las instituciones, ayudando a determinar cuál es la participación de cada uno de los actores escolares en los procesos educativos, que tienen lugar en los centros de trabajo.

Se dirigieron a informantes clave, como son los directores de las 3 escuelas seleccionadas, se realizó también una entrevista del tipo individual, en donde, gracias a su flexibilidad, fue posible ir conduciendo la conversación hacia los puntos de mayor interés, dando oportunidad al entrevistado de explayarse cuando se considerase necesario; lo anterior fue posible, ya que "este tipo de entrevista tiene un guion en el cual se recogen todos los temas que se deben de tratar en el desarrollo de la entrevista.
Las entrevistas grabadas fueron posteriormente transcritas y anotadas para su análisis estableciendo la relación con los resultados y la experiencia reciente de tres escuelas de educación primaria pública en la Ciudad de Puebla.

\section{Resultados}

El propósito es fortalecer la educación en el país, contando con la enorme posibilidad de crecimiento, de mejora, de capacitación para los profesores, de inclusión de valores, invitando a los entrevistados a reflexionar sobre los siguientes factores que ayudarían en la educación integral del alumno:1 Ambiente de la Escuela y del Aula, 2 Compromiso y Trabajo en Equipo de los Docentes, 3 Liderazgo y Gestión, 4 Expectativas del Profesor y de los estudiantes, 5 Las familias y su participación, 6 Los procesos de enseñanza, 7 Instalaciones y Recursos para fortalecer la enseñanza - aprendizaje de los pequeños estudiantes. El papel del contexto del sistema educativo es fundamental en el inicio de experiencias de mejora, propiciándolas con un marco legal adecuado y acciones favorecedoras del cambio.

En los estudios resultó, que en grupos donde los alumnos son desafiados y tienen el apoyo de su profesor, el adelanto era mayor, especialmente por la importancia de que los maestros usaran más preguntas y afirmaciones, estimulando a los alumnos a "usar su imaginación creativa para resolver problemas". Los docentes con una actitud más favorable hacia las innovaciones, al desarrollo profesional, a la formación permanente, la 
autoevaluación como una estrategia de reflexión y mejora, además de su capacidad para integrar nuevas ideas en su propia práctica, su ausentismo, la rotación laboral, entre otros, son aspectos que influyen de forma importante en el éxito de los estudiantes y sus actitudes, si el docente es poco entusiasta sobre el tema o lección que enseña es probable que contagie este sentimiento en los estudiantes lo anterior forma parte de programas más amplios de reforma, investigación o planes de fortalecimiento. Los entrevistados reconocen un agente externo central, los medios de comunicación que desinforman y desacreditan su trabajo y se expresaron de la siguiente manera:

Tenemos que existe presión externa, los medios de comunicación nos atacan sin saber lo que estamos trabajando, que hacemos, lo que más les convence a los padres son los adelantos de los niños. Hay padres de familia que nos presionan, y hay muchos que no se involucran porque trabajan todo el día, el niño viene con su llavecita colgada en el cuello y atiende a sus hermanos más pequeños, eso está en contra del aprendizaje. La mayoría de las mujeres son madres solteras.

Las escuelas están en constante supervisión, han tenido muchas visitas de revisión, por la jefatura de sección,

La Directora de la Escuela 1 indica que experimentan mucha presión por parte de las autoridades federales y estatales, así como de los medios de comunicación. Adicionalmente es interesante notar que pocos padres de familia se preocupan por el desarrollo de sus hijos, existe un bajo nivel de exigencia hacia la escuela. Esto es paradójico, porque por un lado en teoría serían el principal actor que presiona desde un entorno hacia la escuela y por el otro en los hechos, son los que no muestran interés. La escuela se siente presionado por las carencias económicas y sociales de la comunidad, y utilizan este factor para justificar su falta de apoyo tanto a la escuela como a sus hijos.

La supervisión y los mismos padres de familia nos exigen sin embargo hay muchos padres que ni se involucran porque trabajan y eso está en contra de la mejora del aprendizaje, la mayoría de las madres son solteras los niños atienden a sus hermanos más pequeños, entonces no tienen tiempo de hacer las tareas y no cumplen con su responsabilidad. Otros actores que no son propiamente externos son los padres de familia. Como trabajadores y como proveedores económicos, a juicio de docentes y directivos el comportamiento y la falta de compromiso de los padres de familia están también determinadas por agentes externos: los empleadores y una sociedad que no cuida a sus ciudadanos.

La investigación aborda una problemática en la que se analiza la información obtenida y la experiencia reciente de tres escuelas públicas de educación primaria los datos obtenidos denotan la falta de fortalecimiento educativo, esto se refleja en el nivel superior como a continuación se cita; en el ciclo 2017-2018 el 74.0 \% del egreso de Bachillerato y preparato- 
rias ingresa al nivel superior teniendo una deserción del 6.8 \%. (SEP 2018) Se han elaborado diagnósticos en las escuelas de nivel superior obteniendo los siguientes datos: En comprensión de lectura bajo rendimiento, Matemáticas y áreas de ciencias deserción o evitan las profesiones relacionadas con ciencias, falta de integración a los equipos de trabajo, les hace falta fortalecer la toma de decisiones, solución problemas complejos, fortalecer la disciplina en general para cumplir con sus responsabilidades y compromisos. Por lo que es necesario implementar un modelo de educación integral en el nivel de estudios básicos donde se enseñe a los niños las estrategias para mejorar el rendimiento de los alumnos y de esta manera existe la posibilidad de que al llegar al nivel superior cuenten con las competencias necesarias:

Partiendo de los intereses de los alumnos se les pregunta qué saben del tema, si no lo recuerdan se recomienda un ejercicio para que lo que tengan presente, van acumulando conocimientos y reforzando aprendizaje.

Planear el trabajo, de tal forma que se siga un cronograma de actividades esto dará como resultado el logro de los objetivos.

A principios del segundo grado en una de las tres escuelas, había 8 niños que no saben leer ni escribir, según la reforma educativa los niños en segundo ya deben saber leer y escribir y aun sin saber pasan a tercero es importante buscar la manera de que el niño este motivado Cada profesor tiene diferentes estrategias y algunos profesores ocupan parte de su recreo para enseñar a leer a los niños. Por otro lado, sin embargo, se puede discernir otra cara del asunto: al asumir de manera muy concreta y operativa la noción de cambio en la escuela, quizá se corra el riesgo de limitar sus alcances, pues como consta en las entrevistas el tipo de asuntos y decisiones abordados en las reuniones de consejo escolar casi siempre son estrictamente operativos e inmediatos, con pocas posibilidades de pensar como colectivo en el rumbo educativo más general de la escuela. En este sentido, aunque los actores se esfuerzan por cumplir con las exigencias de la política federal, se encuentran relativamente aislados de los objetivos más generales de mejora de la calidad pues no perciben o no utilizan apoyos externos, ya sea de los resultados de las evaluaciones o de la administración educativa estatal, para pensar más a fondo la problemática de sus escuelas y actuar con una visión más estratégica.

Por lo tanto, con la investigación se analizarán las estrategias de la política educativa que tienden a rediseñar los sistemas educativos, tanto centralizadoras como descentralizadoras, o que intentan compensar las condiciones socioeconómicas de los alumnos y favorecer sus aprendizajes, con un modelo vertical, que pocas veces impactan a las escuelas.

El principal referente de la reforma educativa que ha tenido lugar durante las últimas décadas se encuentra en la Declaración Mundial sobre Educación para Todos: Satisfacción de las 
Necesidades Básicas de Aprendizaje. De acuerdo con Castelán (2003b), en esta declaración se establece que los cambios educativos deben darse en un marco de transformación institucional, la cual se logrará teniendo como camino la gestión del sistema. Por lo tanto, el primer paso hacia ese destino fue la implementación de políticas de descentralización, las cuales promueven ajustes en los aspectos administrativos, pedagógicos, de evaluación, comunicación y financiamiento.

Se trataba de reformar una problemática añeja, ya que históricamente el sistema educativo mexicano funcionó con un patrón centralizado que limitó la capacidad de las escuelas para entrar en un ciclo de mejoramiento continuo que estuviese firmemente anclado en la comunidad y la cultura escolar.

Así, en la actualidad un foco importante y persistente de las políticas educativas es la escuela misma, sus formas de gestión, planeación y participación expresada en el lema "La escuela al Centro" en el actual Modelo Educativo (SEP, 2016). Centralizar las decisiones educativas en el nivel federal y buscar descentralizar la actuación es las unidades escolares es un dilema de difícil resolución pues requiere de cambios tanto a nivel del sistema como de la propia institución escolar y se reconoce además que debe ser un proceso de atención permanente, si ha de ir modificando las pautas largamente institucionalizadas en la educación mexicana. El modelo integral de educación consiste en involucrar a todos los actores que participan directa e indirectamente en la educación de los niños y de los jóvenes.

\section{Modelo de Educación Integral}

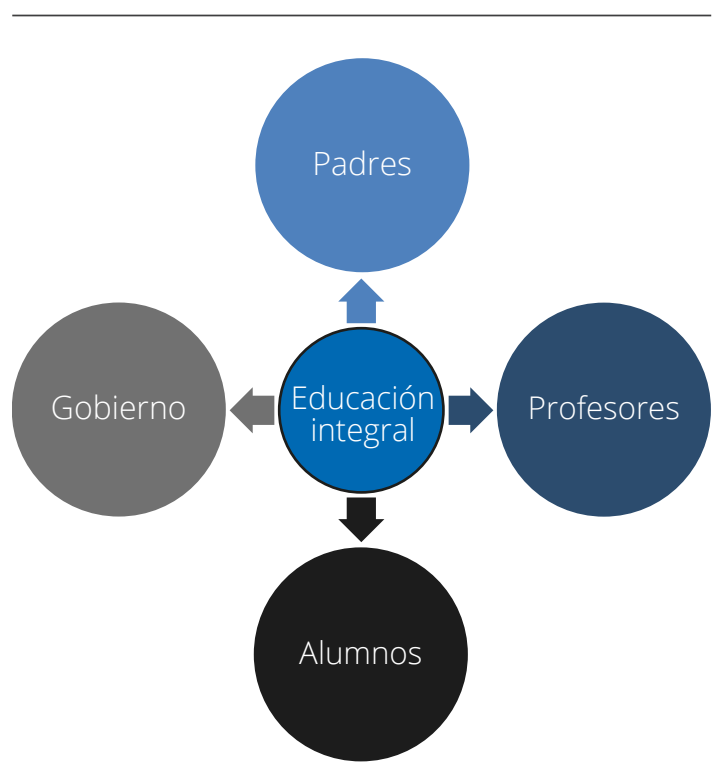

Fuente: Elaboración Propia

\section{Conclusiones}

La Secretaria de educación publica es responsable del funcionamiento del sistema educativo, y todos los que conforman dicha comunidad tienen un compromiso con la mejora de la educación. Las acciones para mejorar la calidad de la educación se dirigen al cumplimiento de los propósitos y objetivos. Para que los objetivos se logren es necesario mejorar los procesos para que se pueda hablar sobre los procesos de calidad, ello implica un reto para la investigación en política educativa, en el contexto de un sistema que está en vías de cambio. Ésta es la perspectiva de la presente investigación.

Por lo anterior se puede concluir lo siguiente: Se observa cómo la SEP ha definido el cambio, ha implementando una ruta de mejora para las escue- 
las, se puede decir que los profesores de estas escuelas están realizando el cambio (tal y como es definido oficialmente). Existen elementos que no juegan a su favor, como el contexto familiar, el papel de las autoridades locales y la evaluación a los profesores. Entonces se puede decir que los maestros y directores de las escuelas hacen lo que pueden con los recursos que tienen a su disposición. Podría, en efecto, ampliar los cambios, pero requerirían de más apoyos institucionales en los tres sectores de gobierno.

La educación integral es aquella que se propone objetivos de aprendizajes relevantes y actualizados, y consigue que los alumnos los alcancen en los tiempos previstos, apoyando en especial a quienes más lo necesiten. La evaluación se concibe como medio indispensable para la mejora continua y el aseguramiento de la calidad, así como para la rendición de cuentas. Además de evaluar, es indispensable dar a conocer los resultados y utilizarlos para la toma de decisiones. El proceso de evaluación y sus resultados deben reconocerse como elementos valiosos que ayuden a escuelas e instituciones a valorar sus logros y limitaciones y a definir y operar innovaciones que les permitan alcanzar niveles superiores de desarrollo y consolidación.

En suma, alcanzar la calidad educativa en las instituciones escolares de cualquier nivel requiere, más que de una buena voluntad, de la interrelación de la gestión educativa con la calidad de los procesos de enseñanza, la conexión con las teorías pedagógicas y del desarrollo humano, la vinculación en el marco de la relación académica con una comunidad en aras de transformar la sociedad y la consecución de recursos tecnológicos y financieros que mejoren cada uno de los procesos. Donde los administradores tienen una tarea relevante por realizar, para lo cual deben prepararse como tales, pues son los gestores de una educación integral. El logro de aprendizajes de calidad es uno de los múltiples retos a los que se enfrentan las comunidades educativas actualmente, lo cual exige hacer énfasis en la formación de los niños, adolescentes, jóvenes y adultos que aspiran a una vida mejor. La educación integral busca formar ciudadanos capaces de comprender las complejas interrelaciones existentes entre ciencia, tecnología y los ámbitos social, económico, político y cultural, con el fin de que, en el futuro, independientemente de su esfera de actividad, cuenten con herramientas imprescindibles para participar en la toma de decisiones que contribuya a construir una sociedad más justa, más sana y más humana. Conjuntar calidad educativa y procesos de gestión, que tributen al mejoramiento del ser humano y de la sociedad, requiere atar fuertemente los pilares de la educación a la vida de los administradores, docentes, padres de familia y los respectivos estudiantes. De esta forma y con estas estrategias también es posible conservar la salud con una alimentación saludable, evitar embarazos no deseados, evitar enfermedades, mejorar el bienestar social y por ende tener mayores oportunidades de lograr objetivos personales y organizacionales para satisfacer las necesidades fundamentales, alcanzando un mejor modo de vida. 


\section{Referencias}

Cárdenas, L. (2004). La teoría de la complejidad y su influencia en la escuela. Revista de Teoría y Didáctica de las Ciencias Sociales, vol- 9, 1.

Cornejo, M. Mendoza (2008), Calidad de la Educación en Iberoamérica: Discursos, políticas y prácticas.

Héctor Monarca (2018) Líderes de la educación superior en Baja California ISBN: 978-84-9148-720-3.

Instituto Nacional de Estadística y Geografía [INEGI]. (2017). Censo Nacional de Población. México: INEGI.

Instituto Nacional para la Evaluación de la Educación [INEE]. (2008). ¿Avanza o retrocede la calidad educativa? Tendencias y perspectivas de la educación básica en México. México: INEE.
Leal Céspedes, J. (2013). El futuro de la Administración - por Juan Carlos Leal Céspedes. Revista GEON (Gestión, Organizaciones Y Negocios), 1(1), 19-22. https://doi. org/10.22579/23463910.137

Murillo, F. (2004). Un marco comprensivo de mejora de la eficacia escolar. Revista Mexicana de Investigación Educativa(21), 319-360.

Posner, C. M. (2004). Enseñanza Efectiva. Revista Mexicana de Investifgación Educativa , 269-276.

Schmelkes, S. (2010). Hacia una mejor calidad de nuestras escuelas.

Torres-Flórez, D. (2019). El entrenamiento del colaborador como estrategia de mejoramiento continuo. Revista GEON (Gestión, Organizaciones Y Negocios), 6(1), 4-9. https://doi. org/10.22579/23463910.149 